\title{
Bounds on crossing symmetry
}

\author{
Sebastian Mizera $\odot^{*}$ \\ Institute for Advanced Study, Einstein Drive, Princeton, New Jersey 08540, USA
}

(Received 23 January 2021; accepted 11 March 2021; published 12 April 2021)

\begin{abstract}
Proposed in 1954 by Gell-Mann, Goldberger, and Thirring, crossing symmetry postulates that particles are indistinguishable from antiparticles traveling back in time. Its elusive proof amounts to demonstrating that scattering matrices in different crossing channels are boundary values of the same analytic function, as a consequence of physical axioms such as causality, locality, or unitarity. In this work we report on the progress in proving crossing symmetry on shell within the framework of perturbative quantum field theory. We derive bounds on internal masses above which scattering amplitudes are crossing symmetric to all loop orders. They are valid for four- and five-point processes, or to all multiplicity if one allows deformations of momenta into higher dimensions at intermediate steps.
\end{abstract}

DOI: 10.1103/PhysRevD.103.L081701

\section{INTRODUCTION}

Ever since its introduction in 1954 by Gell-Mann, Goldberger, and Thirring, crossing symmetry has been widely believed to be a fundamental property of nature [1-3]. It postulates that particles are equivalent to antiparticles with opposite energies and momenta, or-more precisely - that their scattering amplitudes can be analytically continued between different crossing channels. It is routinely taken as an assumption in various bootstrap approaches to the scattering matrix theory; see, e.g., [4-13]. Yet, crossing symmetry does not directly follow from any physical principle and there is only a limited amount of theoretical evidence that it holds in the Standard Model or even a generic quantum field theory. For instance, crossing between $2 \rightarrow 3$ and $3 \rightarrow 2$ processes has never been proven, and neither has any case involving massless particles.

The extent to which we can be certain that crossing symmetry is true nonperturbatively stems from the work of Bros, Epstein, and Glaser, who proved it in the case of $2 \rightarrow 2[14,15]$ and $2 \rightarrow 3[16,17]$ scattering in local quantum field theories with a mass gap, hinging on the assumptions of causality and unitary. Since within the Lehmann-Symanzik-Zimmermann formalism scattering amplitudes do not converge on shell, one is forced to consider off-shell Green's functions, which can be defined in a certain region of the complexified momentum

\footnotetext{
*smizera@ias.edu
}

Published by the American Physical Society under the terms of the Creative Commons Attribution 4.0 International license. Further distribution of this work must maintain attribution to the author(s) and the published article's title, journal citation, and DOI. Funded by SCOAP. space [18-22]. We briefly review this point in Appendix A of [23]. At this stage one is tasked with a purely geometric problem of showing that the envelope of holomorphy of this domain intersects physical regions in all crossing channels on the correct sheet, e.g., using versions of the edge-of-the-wedge theorem. The connection between scattering amplitudes in different channels is achieved via a complex kinematic region of large centerof-mass energy. For reviews see [24-27]. Such proofs are prohibitively long and technical [14-17], and while in principle there is no obstruction to attempting generalizations to higher-point cases, they would certainly not improve our physical understanding of crossing symmetry.

In view of these difficulties, Witten proposed to prove crossing symmetry entirely on shell in perturbation theory, where one might reasonably hope for a simpler and more physical derivation that could potentially extend to higher multiplicity. While work on this problem is ongoing and will be published elsewhere, the purpose of this letter is to demonstrate that even using simple arguments one can put $\mathcal{O}(1)$ bounds on the ratios of masses above which crossing symmetry is satisfied to all loop orders.

Since for a $C P T$-invariant theory crossing is already apparent on the level of Feynman diagrams, the challenge lies in showing that Feynman integrals cannot develop singularities when continued between any pair of crossed processes. To make the problem well defined we assume that any overall divergences (such as infrared or ultraviolet), if present, have been regularized or renormalized. As a consequence, one has to consider scalar diagrams of all graph topologies with an arbitrary number of loops and external legs $n$. To each of them we can assign the function

$$
\mathcal{V}=\sum_{e} \alpha_{e}\left(q_{e}^{2}-m_{e}^{2}\right)
$$


which can be thought of as the localized worldline action. Here $\alpha_{e}$ are the Schwinger proper times associated to the internal edges $e$, while $q_{e}^{\mu}$ and $m_{e}$ are the momenta and masses flowing through them, respectively, sourced by the external momenta $p_{i}^{\mu}$. Due to homogeneity in $\alpha_{e}$ 's, extremizing the action requires $\mathcal{V}=0$, which is a necessary condition for a singularity, equivalent to putting propagators on shell.

Were it not for the requirement of causality, scattering amplitudes would be analytic along complex paths connecting any two real nonsingular points in the space of kinematic invariants $p_{i} \cdot p_{j}$, because along such a deformation

$$
\sum_{e} \alpha_{e}\left|q_{e}^{2}-m_{e}^{2}\right|^{2}>0
$$

ergo, it is impossible to simultaneously put all propagators on shell. However, such analytic continuations generically violate causality, which requires that $\operatorname{Im} \mathcal{V}>0$ when approaching physical points, as dictated by the $i \varepsilon$ prescription. Its consistent implementation is what puts bounds on crossing symmetry.

One way of ensuring causality is analytic continuation via a region where $\mathcal{V}<0$ for all values of Schwinger parameters. We will show it implies that the internal masses $m_{e}$ cannot be too light, or more precisely

$$
m_{e}>\frac{\sqrt{n}}{2 \sqrt{2}} \sqrt{\max _{i}\left(M_{i}^{2}, \frac{\sum_{j} M_{j}^{2}-2 M_{i}^{2}}{n-2}\right)},
$$

where $M_{i}$ are the external masses. These are bounds for crossing symmetry to be satisfied on shell to all loop orders in perturbation theory.

For instance, the above bounds are satisfied for scattering of massless particles with all the exchanged states having arbitrary nonzero mass. This result implies crossing symmetry for a range of low-energy effective field theories, which at present does not have a counterpart on the nonperturbative level $[15,17]$.

For $n=4,5$ in the equal mass case, $M_{i}=M$, we have, respectively,

$$
m_{e} \gtrsim 0.707 M, \quad m_{e} \gtrsim 0.791 M,
$$

and for scattering of the lightest states, i.e., $m_{e} \geq M$, crossing symmetry is valid for $n<8$. A closely related kinematic region was previously investigated in the context of dispersion relations, majorization of Feynman diagrams, and related topics for $n=4$ with the same bound in the equal-mass case [31], and for $n=5$ [31-34] without attempts to put bounds.

The above strategy relies on linear deformations of the kinematic invariants $p_{i} \cdot p_{j}$ rather than the momenta $p_{i}^{\mu}$ themselves. The advantage of doing so is that we can continue between crossed processes involving a different number of incoming or outgoing particles, such as a continuation from $2 \rightarrow 3$ to $3 \rightarrow 2$ scattering. A disadvantage of this approach lies in the fact that along the deformation the momenta $p_{i}^{\mu}$ will in general span an $(n-1)$-dimensional space. Hence for $n \geq 6$ the proof requires deformations of momenta into higher dimensions at intermediate steps. While it certainly makes sense when we treat scattering amplitudes as functions of complex variables, the physical interpretation is obscured.

We work in Minkowski space with mostly minus signature and use conventions where incoming momenta are denoted with $p_{i}^{\mu}$ and outgoing $-p_{i}^{\mu}$, such that the momentum conservation reads $\sum_{i} p_{i}^{\mu}=0$.

\section{REVIEW OF FEYNMAN INTEGRALS AND THEIR SINGULARITIES}

We find it most intuitive to interpret Feynman diagrams in the worldline formalism, where Schwinger proper times $\alpha_{e}$ are the only dynamical variables. A scalar diagram with $n \geq 4$ external legs, E internal edges (propagators), and $\mathrm{L}$ loops in $\mathrm{D}$ space-time dimensions can be written as

$$
\int_{0}^{\infty} \frac{\mathrm{d}^{\mathrm{E}} \alpha_{e}}{\mathcal{U}^{\mathrm{D} / 2}} e^{i \mathcal{V} / \hbar}
$$

where $\mathcal{V}$ is the localized action and $\mathcal{U}$ is the determinant of the Laplacian of the diagram. For real kinematics, it is then customary to use the rescaling invariance $\alpha_{e} \rightarrow \lambda \alpha_{e}$ to integrate out the overall scale $\lambda$, which gives up to normalization

$$
\int_{0}^{1} \frac{\mathrm{d}^{\mathrm{E}} \alpha_{e} \delta\left(\sum_{e} \alpha_{e}-1\right)}{\mathcal{U}^{\mathrm{D} / 2} \mathcal{V}^{\mathrm{E}-\mathrm{LD} / 2}},
$$

where $\mathrm{E}-\mathrm{LD} / 2$ is the superficial degree of divergence. It is understood that causality and convergence are imposed either by shifting $\mathcal{V} \rightarrow \mathcal{V}+i \varepsilon$ with infinitesimal $\varepsilon$, or as a contour deformation; see Appendix B of [23]. Here we will not deform $\alpha_{e}$ 's, but instead implement causality by deforming the external kinematics such that $\operatorname{Im} \mathcal{V}>0$ when a physical limit is approached. We complexify kinematics only after the representation (6) is obtained.

We will assume that the Feynman integral is free of overall divergences, which can be dealt with (for example in dimensional regularization) without affecting the singularities of the integral. Note that if a renormalization scheme is employed, $m_{e}$ 's denote the renormalized masses which makes the bounds on masses (3) a scheme-dependent statement. We have to consider only diagrams dependent on at least one kinematic invariant, since others are either finite or excluded by the above assumption. Without loss of generality we consider oneparticle irreducible diagrams from now on, since inclusion 
of reducible diagrams can at most introduce simple poles that do not affect the analytic continuation described below.

All quantities in (6) can be expressed in terms of combinatorics of the Feynman diagram; see, e.g., [35]. The action can be written as (sums in this letter always range in $i, j=1,2, \ldots, n$ and $e=1,2, \ldots, \mathrm{E})$ :

$$
\mathcal{V}:=-\sum_{i<j} p_{i} \cdot p_{j} \mathcal{G}_{i j}-\sum_{e} m_{e}^{2} \alpha_{e},
$$

where $m_{e}$ is the mass associated to the edge $e$ and $\mathcal{G}_{i j}$ is the Green's function (for a scalar field on a graph) between vertices where $p_{i}^{\mu}$ and $p_{j}^{\mu}$ enter the Feynman diagram. The graph Green's function $\mathcal{G}_{i j}$ measures the response of the diagram to changes in the dot product $p_{i} \cdot p_{j}$ and does not depend on $n$ but only on the topology of the diagram. This representation makes it obvious that we are dealing with a superposition of multiple two-point function problems, all jumbled up due to the momentum conservation [36].

For completeness we give expressions for $\mathcal{U}$ and $\mathcal{G}_{i j}$, though they will not be needed directly in the proof. The polynomial $\mathcal{U}$ is given by

$$
\mathcal{U}:=\sum_{T} \prod_{e \notin T} \alpha_{e}
$$

where the sum is over all spanning trees $T$ obtained by removing exactly $L$ edges from the diagram. The individual $\mathcal{G}_{i j}$ 's can be written as

$$
\mathcal{G}_{i j}:=\frac{1}{\mathcal{U}} \sum_{F_{i j}} \prod_{e \notin F_{i j}} \alpha_{e},
$$

which sums over all spanning two-forests $F_{i j}=T_{i} \sqcup T_{j}$, obtained by cutting $\mathrm{L}+1$ edges such that vertices where $p_{i}^{\mu}$ and $p_{j}^{\mu}$ enter the diagram belong to (possibly empty) trees $T_{i}$ and $T_{j}$, respectively. An example is given in Appendix C of [23].

\section{A. Landau equations}

Singularities of Feynman integrals are governed by Landau equations [38], which in the representation (6) $\operatorname{read}[39,40]$

$$
\alpha_{e} \frac{\partial \mathcal{V}}{\partial \alpha_{e}}=0
$$

for all edges $e$. Since a solution involving $\alpha_{e^{\prime}}=0$ gives Landau equations for a simpler graph with the edge $e^{\prime}$ contracted, and we already take into account all graph topologies, we only need to consider leading Landau equations with $\alpha_{e} \neq 0$ (in other words, the analytic continuation we will employ avoids subleading Landau singularities just as well as the leading ones). One can interpret them as the classical limit of the action $\mathcal{V}$ where all propagators go on shell according to (1). See Appendix D of [23] for more details [41]. Recent work on Landau equations includes [43-47].

Given the definition in (7), $\mathcal{V}$ is a degree-one homogeneous function in $\alpha_{e}$ 's, which means on the solution of Landau equations we have

$$
\mathcal{V}=\sum_{e} \alpha_{e} \frac{\partial \mathcal{V}}{\partial \alpha_{e}}=0
$$

This is a necessary (but not sufficient) condition for a singularity. Since leading Landau equations require $\alpha_{e}>0$, the definitions (8) and (9) give $\mathcal{U}>0$ and $\mathcal{G}_{i j}>0$.

\section{B. Upper bound on the graph Green's functions}

In the following steps we will need an upper bound on $\mathcal{G}_{i j}$ that does not depend on the number of loops, edges, or external states. As a proxy for its derivation, let us briefly consider the case $n=2$ off shell, where $-p_{1} \cdot p_{2}=p_{1}^{2}$ is allowed to vary and anomalous thresholds are absent. We have

$$
\mathcal{V}=p_{1}^{2} \mathcal{G}_{12}-\sum_{e} m_{e}^{2} \alpha_{e} \leq p_{1}^{2} \mathcal{G}_{12}-m^{2},
$$

where $m$ is the lightest of $m_{e}>0$ and we used $\sum_{e} \alpha_{e}=1$. Since $\mathcal{V}<0$ for $p_{1}^{2}=0$, the action has to stay negative before encountering the first physical threshold at

$$
p_{1}^{2}=\left(\sum_{e \in R} m_{e}\right)^{2} \geq|R|^{2} m^{2},
$$

where $R$ is the set of $|R|$ intermediate particles, as in Fig. 1. This implies $\mathcal{G}_{12} \leq 1 /|R|^{2}$. Since the labeling of the momenta was arbitrary, we have

$$
\mathcal{G}_{i j} \leq \frac{1}{4}
$$

because $|R| \geq 2$ for one-particle irreducible diagrams. An alternative derivation using only graph theory is given in [48], which shows it holds without any restriction on masses.

We remind the reader that analyticity of higher-point on-shell amplitudes is not well understood because of the presence of anomalous thresholds. The above trick circumvents this issue by deriving bounds on the individual

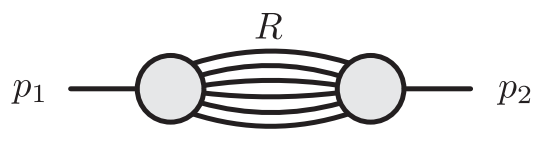

FIG. 1. Normal threshold for $n=2$, where a subset $R$ of propagators goes on shell. 
$\mathcal{G}_{i j}$ which are the building blocks entering (7) for arbitrary $n$.

\section{BOUNDS ON CROSSING SYMMETRY}

We will show how to analytically continue between two nonsingular points in the real kinematic space, denoted by $p_{i}^{(0)} \cdot p_{j}^{(0)}$ and $p_{i}^{(1)} \cdot p_{j}^{(1)}$, i.e., where Landau equations are not satisfied. The deformation takes place in the $n(n-3) / 2$-dimensional space of independent kinematic invariants $p_{i} \cdot p_{j}$, whose preimage in the momentum vectors $p_{i}^{\mu}$ can be only realized in an $(n-1)$-dimensional space in the intermediate steps.

We preface the discussion with a naive approach in order to highlight why it is causality that puts bounds on crossing symmetry.

\section{A. Naive approach}

We introduce a complex variable $z$ and linearly deform the kinematic invariants according to

$$
p_{i} \cdot p_{j}=p_{i}^{(0)} \cdot p_{j}^{(0)}+z\left(p_{i}^{(1)} \cdot p_{j}^{(1)}-p_{i}^{(0)} \cdot p_{j}^{(0)}\right),
$$

as well as consider a path in the upper-half plane approaching the two kinematic points at $z=0$ and $z=1$; see Fig. 2. This deformation preserves momentum conservation and on-shell conditions, $p_{i}^{2}=M_{i}^{2}$.

Since $\mathcal{V}$ responds linearly to changes in kinematics, we have

$$
\mathcal{V}=\mathcal{V}_{0}+z\left(\mathcal{V}_{1}-\mathcal{V}_{0}\right)
$$

where $\mathcal{V}_{\alpha}:=\mathcal{V}\left(p_{i}^{(\alpha)} \cdot p_{j}^{(\alpha)}\right)$. Remaining on the original integration contour with $\alpha_{e}$ real, the real and imaginary parts of the leading Landau equations are, respectively,

$$
\begin{gathered}
\frac{\partial \mathcal{V}_{0}}{\partial \alpha_{e}}+\operatorname{Re} z\left(\frac{\partial \mathcal{V}_{1}}{\partial \alpha_{e}}-\frac{\partial \mathcal{V}_{0}}{\partial \alpha_{e}}\right)=0, \\
\operatorname{Im} z\left(\frac{\partial \mathcal{V}_{1}}{\partial \alpha_{e}}-\frac{\partial \mathcal{V}_{0}}{\partial \alpha_{e}}\right)=0,
\end{gathered}
$$

for all edges $e$. These cannot be simultaneously satisfied along the deformation path with $\operatorname{Im} z>0$ : vanishing of the imaginary part implies $\partial \mathcal{V}_{0} / \partial \alpha_{e}=0$ for all $e$, which is

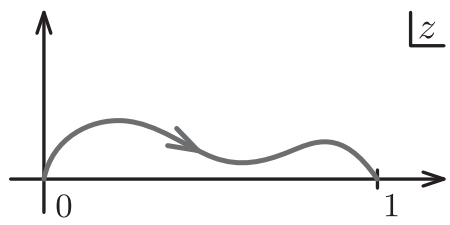

FIG. 2. Path of deformation in the upper-half plane. a contradiction. Hence there are no singularities in the upper-half plane of $z$.

Nevertheless, this deformation cannot be used because by utilizing the imaginary part of $\mathcal{V}$ for the deformation we lost a reliable way of imposing the $i \varepsilon$ prescription near both of two physical points. Put differently, the path of analytic continuation will in general veer away from the physical sheet.

An exception to this point are planar diagrams, which have vastly simpler analyticity properties and crossing for $n \leq \mathrm{D}+1$ can be proven without any constraints on masses; see Appendix E of [23].

\section{B. Fixing the $i \varepsilon$}

In order to guarantee the correct $i \varepsilon$ prescription we will add an intermediate step in the deformation, which passes through an open set $\left\{p_{i}^{(*)} \cdot p_{j}^{(*)}\right\}$ for which

$$
\mathcal{V}_{*}<0
$$

across the whole integration contour. For the time being let us assume such points exist and deform

$$
p_{i} \cdot p_{j}=p_{i}^{(*)} \cdot p_{j}^{(*)}+z\left(p_{i}^{(1)} \cdot p_{j}^{(1)}-p_{i}^{(*)} \cdot p_{j}^{(*)}\right)
$$

followed by an analogous continuation connecting $p_{i}^{(*)}$. $p_{j}^{(*)}$ to $p_{i}^{(0)} \cdot p_{j}^{(0)}$. We further restrict to $\operatorname{Re} z \geq 0$. There are two cases depending on the sign of $\mathcal{V}_{1}-\mathcal{V}_{*}$. When $\mathcal{V}_{1}>\mathcal{V}_{*}$ we have

$$
\operatorname{Im} \mathcal{V}=\operatorname{Im} z\left(\mathcal{V}_{1}-\mathcal{V}_{*}\right)>0
$$

which is the correct causal prescription. Otherwise, when $\mathcal{V}_{1} \leq \mathcal{V}_{*}$, we have

$$
\operatorname{Re} \mathcal{V}=\mathcal{V}_{*}+\operatorname{Re} z\left(\mathcal{V}_{1}-\mathcal{V}_{*}\right)<0,
$$

since $\mathcal{V}_{*}<0$ by assumption. In those cases there is no singularity on the real axis and hence the $i \varepsilon$ is not needed.

It remains to prove that a set of $\left\{p_{i}^{(*)} \cdot p_{j}^{(*)}\right\}$ with $\mathcal{V}_{*}<0$ exists in the first place.

\section{Bounds on masses}

We consider kinematics with $-p_{i}^{(*)} \cdot p_{j}^{(*)}<c$ for each of the $n(n-1) / 2$ kinematic invariants appearing in $\mathcal{V}_{*}$ and some positive constant $c$. Using the upper bound on $\mathcal{G}_{i j}$ from (14) and calling $m=\min _{e}\left(m_{e}\right)$ the lightest internal mass one finds

$$
\mathcal{V}_{*}<\frac{n(n-1)}{8} c-m^{2}
$$

Therefore, requiring that $\mathcal{V}_{*}<0$ yields 


$$
-p_{i}^{(*)} \cdot p_{j}^{(*)}<c<\frac{8}{n(n-1)} m^{2} .
$$

In terms of the external masses $M_{i}$ this translates to two types of constraints. Using momentum conservation requires on the one hand

$$
M_{i}^{2}=-p_{i}^{(*)} \cdot \sum_{j \neq i} p_{j}^{(*)}<\frac{8}{n} m^{2}
$$

and on the other

$$
\sum_{j} M_{j}^{2}-2 M_{i}^{2}=-\sum_{j \neq i} p_{j}^{(*)} \cdot \sum_{k \neq i, j} p_{k}^{(*)}<\frac{8(n-2)}{n} m^{2}
$$

for all $i$. They together imply the constraints (3). These are the conditions for crossing symmetry to be satisfied to all loops and multiplicities.

\section{OUTLOOK}

Let us comment on two natural directions for future work: optimizing the bounds and preventing the momenta $p_{i}^{\mu}$ from wandering into higher dimensions.

With respect to the former, let us notice that the upper bounds $\mathcal{G}_{i j} \leq 1 /|R|^{2}$ are saturated on configurations where $\alpha_{e} \approx 1 /|R|$ for each of the intermediate lines $e \in R$, and $\alpha_{e} \approx 0$ otherwise; cf. Fig. 1. Clearly, such bounds cannot be attained for all $\mathcal{G}_{i j}$ 's simultaneously, because $\mathcal{G}_{i j}$ are not mutually independent (for example, they satisfy $\mathcal{G}_{i j}+\mathcal{G}_{j k} \geq \mathcal{G}_{i k}$ ). It is not unlikely that exploiting such interdependencies can improve bounds on crossing symmetry, though probably not significantly so for generic quantum field theories. On the other hand, implementing conservation laws for specific processes might improve the bounds, perhaps along the lines of previous work on dispersion relations [49,50].

Remaining in four dimensions for $\operatorname{Im} z>0$ requires imposing vanishing of every $5 \times 5$ minor of $p_{i} \cdot p_{j}$ treated as a matrix, which would violate linear dependence on $z$ that our arguments hinged upon. Instead, one should employ a deformation directly on the four-momenta $p_{i}^{\mu}$ that correspond to linear shifts of $p_{i} \cdot p_{j}$, such as those used in on-shell recursion relations [51]. Nonetheless, in some situations it might be possible to get away without doing so, such as in the case of four-point scattering in two dimensions with equal external masses, $M_{i}=M$. In this setup we have $\left(p_{1}+p_{3}\right)^{2}=0$ and repeating the steps from previous sections gives

$$
\mathcal{V}_{*} \leq \frac{1}{2}\left(s_{*}+M^{2}\right)-m^{2}
$$

with $s_{*}=\left(p_{1}^{(*)}+p_{2}^{(*)}\right)^{2}$. Since we can choose $s_{*}$ to be arbitrarily small, it guarantees crossing symmetry and maximal analyticity for $m_{e}>M / \sqrt{2}$ to all loops. Exceptions to this statement are scattering matrices of integrable theories, which cannot be represented as boundary values of a single analytic function (see, e.g., [52]), reflecting vanishing probability for particle production and hence breakdown of crossing symmetry.

\section{ACKNOWLEDGMENTS}

The author thanks Edward Witten for illuminating discussions. He gratefully acknowledges the funding provided by Frank and Peggy Taplin as well as Grant No. DESC0009988 from the U.S. Department of Energy.
[1] M. Gell-Mann, M. Goldberger, and W. E. Thirring, Use of causality conditions in quantum theory, Phys. Rev. 95, 1612 (1954).

[2] M. Gell-Mann and M. Goldberger, Scattering of low-energy photons by particles of spin 1/2, Phys. Rev. 96, 1433 (1954).

[3] M. Gell-Mann, We never wrote up the crossing relations, Web of Stories.

[4] D. Atkinson, A proof of the existence of functions that satisfy exactly both crossing and unitarity: I. Neutral pionpion scattering. No subtractions, Nucl. Phys. B7, 375 (1968); Erratum, Nucl. Phys. B15, 331 (1970).

[5] A. Martin, Scattering Theory: Unitarity, Analyticity and Crossing (Springer, Berlin, Heidelberg, 1969), Vol. 3, https://doi.org/10.1007/BFb0101043.

[6] A. Adams, N. Arkani-Hamed, S. Dubovsky, A. Nicolis, and R. Rattazzi, Causality, analyticity and an IR obstruction to UV completion, J. High Energy Phys. 10 (2006) 014.
[7] M. F. Paulos, J. Penedones, J. Toledo, B. C. van Rees, and P. Vieira, The S-matrix bootstrap. Part III: Higher dimensional amplitudes, J. High Energy Phys. 12 (2019) 040.

[8] A. L. Guerrieri, J. Penedones, and P. Vieira, Bootstrapping QCD Using Pion Scattering Amplitudes, Phys. Rev. Lett. 122, 241604 (2019).

[9] B. Bellazzini, J. Elias Miró, R. Rattazzi, M. Riembau, and F. Riva, Positive moments for scattering amplitudes, arXiv: 2011.00037.

[10] A. J. Tolley, Z.-Y. Wang, and S.-Y. Zhou, New positivity bounds from full crossing symmetry, arXiv:2011.02400.

[11] A. Guerrieri, J. Penedones, and P. Vieira, S-matrix bootstrap for effective field theories: Massless pions, arXiv:2011.02802.

[12] S. Caron-Huot and V. Van Duong, Extremal effective field theories, arXiv:2011.02957.

[13] P. Tourkine and A. Zhiboedov, Scattering from production in $2 \mathrm{~d}$, arXiv:2101.05211. 
[14] J. Bros, H. Epstein, and V. J. Glaser, Some rigorous analyticity properties of the four-point function in momentum space, Nuovo Cimento 31, 1265 (1964).

[15] J. Bros, H. Epstein, and V. Glaser, A proof of the crossing property for two-particle amplitudes in general quantum field theory, Commun. Math. Phys. 1, 240 (1965).

[16] J. Bros, V. Glaser, and H. Epstein, Local analyticity properties of the $n$ particle scattering amplitude, Helv. Phys. Acta 45, 149 (1972).

[17] J. Bros, Derivation of asymptotic crossing domains for multiparticle processes in axiomatic quantum field theory: A general approach and a complete proof for $2 \rightarrow 3$ particle processes, Phys. Rep. 134, 325 (1986).

[18] O. Steinmann, Über den Zusammenhang zwischen den Wightmanfunktionen und den retardierten Kommutatoren, Helv. Phys. Acta 33, 257 (1960).

[19] O. Steinmann, Wightman-Funktionen und retardierte Kommutatoren. II, Helv. Phys. Acta 33, 347 (1960).

[20] D. Ruelle, Connection between Wightman functions and Green functions in p-space, Nuovo Cimento 19, 356 (1961).

[21] H. Araki, Generalized retarded functions and analytic function in momentum space in quantum field theory, J. Math. Phys. (N.Y.) 2, 163 (1961).

[22] H. Araki and N. Burgoyne, Properties of the momentum space analytic function, Nuovo Cimento 18, 342 (1960).

[23] See Supplemental Material at http://link.aps.org/ supplemental/10.1103/PhysRevD.103.L081701 for a review of previous results and examples.

[24] H. Epstein, Some analytic properties of scattering amplitudes in quantum field theory, in Proceedings of the 8th Brandeis University Summer Institute in Theoretical Physics: Particle Symmetries and Axiomatic Field Theory (Gordon and Breach, Science Publishers, Inc., New York, 1966), pp. 1-128.

[25] G. Sommer, Present state of rigorous analytic properties of scattering amplitudes, Fortschr. Phys. 18, 577 (1970).

[26] N. Bogolubov, A. Logunov, A. Oksak, and I. Todorov, General Principles of Quantum Field Theory, Mathematical Physics and Applied Mathematics (Springer, Netherlands, 1989).

[27] See also [28] for progress using the assumption of asymptotic completeness and [29,30] for reviews of previous incomplete attempts at proving crossing symmetry.

[28] J. Bros, Analytic structure of Green's functions in quantum field theory, in Mathematical Problems in Theoretical Physics, edited by K. Osterwalder (Springer, Berlin, 1980), pp. 166-199.

[29] R. J. Eden, P. V. Landshoff, D. I. Olive, and J. C. Polkinghorne, The Analytic S-Matrix (Cambridge University Press, Cambridge, England, 1966), Sec. IV.8.

[30] D. Iagolnitzer, The S Matrix (North-Holland Publishing Company, Amsterdam, 1978), App. IV.

[31] T. T. Wu, Domains of definition for Feynman integrals over real Feynman parameters, Phys. Rev. 123, 678 (1961).

[32] L. F. Cook and J. Tarski, Some properties of the five-point function in perturbation theory, J. Math. Phys. (N.Y.) 3, 1 (1962).

[33] D. Branson, P. V. Landshoff, and J. C. Taylor, Derivation of partial amplitudes and the validity of dispersion relations for production processes, Phys. Rev. 132, 902 (1963).
[34] J. B. Boyling, Hermitian analyticity and extended unitarity in S-matrix theory, Nuovo Cimento 33, 1356 (1964).

[35] N. Nakanishi, Graph Theory and Feynman Integrals, Mathematics and its applications: A series of monographs and texts (Gordon and Breach, New York, 1971).

[36] The representation (7) makes it transparent why proving crossing symmetry off shell is trivial in perturbation theory: all the $n(n-1) / 2$ kinematic invariants can be deformed independently in $\operatorname{Im} p_{i} \cdot p_{j}<0$ for $i \neq j$ while preserving analyticity and causality (see also [37] for the loop momentum perspective). It is imposing on shellness, i.e., fixed $p_{i}^{2}=M_{i}^{2}$, that introduces difficulties.

[37] C. De Lacroix, H. Erbin, and A. Sen, Analyticity and crossing symmetry of superstring loop amplitudes, J. High Energy Phys. 05 (2019) 139.

[38] L. Landau, On analytic properties of vertex parts in quantum field theory, Nucl. Phys. 13, 181 (1960).

[39] N. Nakanishi, Ordinary and anomalous thresholds in perturbation theory, Prog. Theor. Phys. 22, 128 (1959).

[40] J. D. Bjorken, Experimental tests of quantum electrodynamics and spectral representations of Green's functions in perturbation theory, Ph.D. thesis, Stanford University, 1959.

[41] In going from (5) to (6) we broke projective invariance, which obscures potential singularities at infinities [42]. They need not concern us because we will not deform the integration contour.

[42] D. B. Fairlie, P. V. Landshoff, J. Nuttall, and J. C. Polkinghorne, Singularities of the second type, J. Math. Phys. (N.Y.) 3, 594 (1962).

[43] P. Chin and E. Tomboulis, Nonlocal vertices and analyticity: Landau equations and general Cutkosky rule, J. High Energy Phys. 06 (2018) 014.

[44] I. Prlina, M. Spradlin, and S. Stanojevic, All-Loop Singularities of Scattering Amplitudes in Massless Planar Theories, Phys. Rev. Lett. 121, 081601 (2018).

[45] J. Collins, A new and complete proof of the Landau condition for pinch singularities of Feynman graphs and other integrals, arXiv:2007.04085.

[46] S. Komatsu, M. F. Paulos, B. C. Van Rees, and X. Zhao, Landau diagrams in AdS and S-matrices from conformal correlators, J. High Energy Phys. 11 (2020) 046.

[47] M. Mühlbauer, Momentum space Landau equations via isotopy techniques, arXiv:2011.10368.

[48] N. Nakanishi, Parametric integral formulas and analytic properties in perturbation theory, Prog. Theor. Phys. Suppl. 18, 1 (1961).

[49] J. Boyling, Majorization of Feynman diagrams in which a heavy particle is conserved, Ann. Phys. (N.Y.) 25, 249 (1963).

[50] J. Boyling, Majorization of Feynman diagrams involving pions and nucleons, Ann. Phys. (N.Y.) 28, 435 (1964).

[51] R. Britto, F. Cachazo, B. Feng, and E. Witten, Direct Proof of Tree-Level Recursion Relation in Yang-Mills Theory, Phys. Rev. Lett. 94, 181602 (2005).

[52] D. Iagolnitzer, Factorization of the multiparticle $S$ matrix in two-dimensional space-time models, Phys. Rev. D 18, 1275 (1978). 